\title{
Management of Primitive Neuroectodermal Tumor of the Kidney with Inferior Vena Cava Thrombus
}

\author{
Sahil Gupta Kaustav Majumder Anurag Chahal Ashish K. Saini Arjun Gupta \\ University of Texas Southwestern Medical Centre, Dallas, Tex., USA
}

\section{Key Words}

Primitive neuroectodermal tumors • Kidney cancer • Immunohistochemistry • Thrombosis

\begin{abstract}
Primitive neuroectodermal tumors (PNET) are an aggressive group of small round cell tumors usually arising in the nervous system and affecting children. They have a tendency for local invasion, distant spread and formation of tumor thrombi. The kidney is a rare primary location for these tumors. Outcomes are frequently poor due to late diagnosis (Wilms tumor is a more common tumor in this population) and early spread. Immunohistochemistry is invaluable in making the diagnosis of PNET. We report a case of a primary renal PNET with extensive tumor thrombus into the inferior vena cava, and lung metastasis in a pediatric patient, and its successful management. Our 14-year-old patient with renal PNET was managed with radical nephrectomy, thrombectomy and chemotherapy and remains disease free to date. The diagnosis of renal PNETs should be considered in young adult patients who present with aggressive renal masses at initial presentations. Despite its aggressive nature, good outcomes can be achieved by a multimodality therapeutic strategy.

Copyright $\odot 2015$ S. Karger AG, Basel
\end{abstract}

\section{KARGER}

Fax +4161306 1234

E-Mailkarger@karger.ch

www.karger.com
(C) 2015 S. Karger AG, Basel

$1015-9770 / 14 / 0091-0047 \$ 38.00 / 0$

Accessible online at:

www.karger.com/cur

\section{Introduction}

Primitive neuroectodermal tumors (PNET) are a group of small round cell tumors derived from the neural crest [1]. PNET can rarely arise outside the nervous system with bones and soft tissue being common extra cranial sites. The kidney is a rare primary location for these tumors [2]. Approximately 50 cases of renal PNET have been reported in the literature, and an accompanying tumor thrombus has been reported very rarely [3-5]. Renal PNET is a highly aggressive cancer usually presenting at an advanced stage with metastasis. It affects young adults with significant mortality owing to the late diagnosis, advanced stage at presentation and aggressive course of the disease $[6,7]$. We report a case of a primary renal PNET with extensive inferior vena cava (IVC) thrombus in a pediatric patient, and its successful management.

\section{Case Report}

A 14-year-old boy presented with right upper quadrant discomfort and 2 episodes of reddish urine over the past month. He had no reported medical issues. He denied any constitutional or lower urinary tract symptoms, and had no family history of renal disease or nephrolithiasis. He reported no recent infections or vaccinations. 

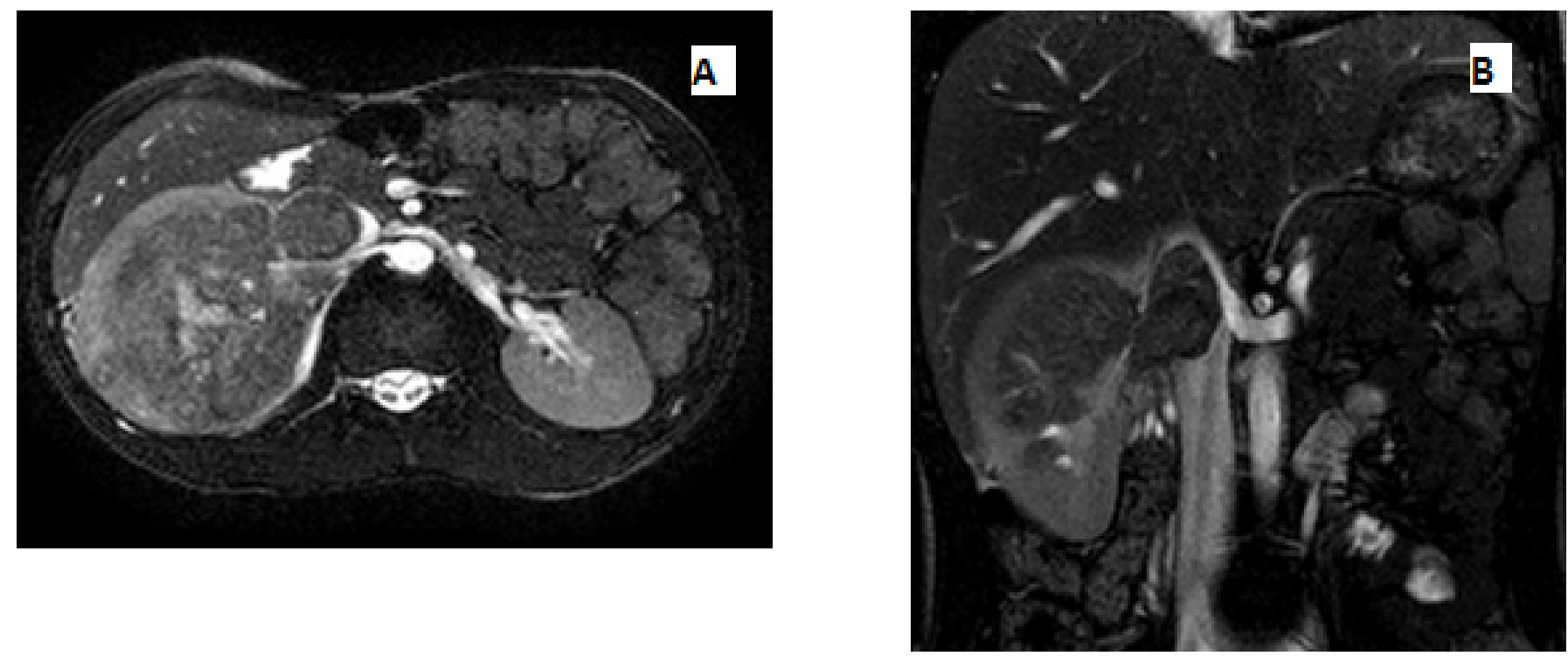

Fig. 1. Renal tumor extending into the renal vein and inferior vena cava seen on MRI (balanced turbo field echo) of the abdomen. A Transverse section; B longitudinal section.

On exam, he was afebrile with pulse rate $82 / \mathrm{min}$, blood pressure $128 / 76 \mathrm{mmHg}$, respiratory rate $13 / \mathrm{min}$. General physical examination was unremarkable. Back was non-tender with negative thump test. On palpation a firm mass was palpated in the right lumbar region below the costal margin, which was ballotable and non-tender with well-defined margins.

Laboratory analysis revealed white blood cell count $8.3 \times$ $10^{9} / 1$, hemoglobin $10.1 \mathrm{~g} / \mathrm{dl}$, platelet count $318 \times 10^{9} / 1$. Urinalysis was unremarkable. Blood urea was $37 \mathrm{mg} / \mathrm{dl}$ and serum creatinine was $0.8 \mathrm{mg} / \mathrm{dl}$.

Ultrasonography of the abdomen demonstrated an enlarged right kidney with a heterogenous mass extending into the right renal vein and IVC. A non contrast MRI demonstrated a $5 \times 4 \times 6$ $\mathrm{cm}$ right sided renal mass with right renal thrombus extending into the IVC up till its infra hepatic course (fig. 1).

The differential diagnosis at this stage included Wilms tumor, renal cell carcinoma, polycystic kidney disease and possibility of a rare tumor.

A decision to perform open right radical nephrectomy along with IVC thrombectomy was taken in view of possibility of thrombus dislodgement. A Chevron incision was given and a $6 \times$ $5 \mathrm{~cm}$ mass was seen in the mid and superior pole of right kidney with multiple enlarged aortocaval lymph nodes. Diffuse desmoplastic reaction was seen around the area of tumor and in aortocaval region. There was tumor in renal vein extending in IVC for $5 \mathrm{~cm}$ from the renal vein ostium. No local invasion was noted. Surgery was well tolerated.

On gross examination, a solid greyish white tumor was identified, measuring $10 \times 8 \times 7 \mathrm{~cm}$, replacing almost the whole renal parenchyma and infiltrating into the renal pelvis. Microscopic examination revealed features of a malignant round cell tumor which showed strong immunopositivity for MIC-2 (CD-99), chromogranin and synaptophysin (fig. 2). Overall features were consistent with a PNET.
Post-operative contrast enhanced CT of thorax, abdomen and pelvis showed no residual disease in the abdomen and pelvis but new bilateral lung nodularities suggestive of bilateral lung metastasis. Chemotherapy with vincristine, adriamycin, cyclophosphamide with alternating ifosfamide and etoposide was initiated. At last follow up 1 year after surgery, the patient is healthy with stable serum creatinine, no clinical complaints and whole body contrast enhanced CT with no evidence of tumor.

\section{Discussion}

PNETs, along with Askin tumor and Ewing sarcoma (ES), exist as members of the ES/PNET family of tumors, which comprises $1 \%$ of all sarcomas [1]. As their name would suggest, PNETs arise from the cells of the primitive neuroectoderm [8].

PNET of the kidney is rare and in it there are only a handful of reported cases of PNET presenting with IVC thrombus. The most common renal neoplasm in childhood is Wilms tumor, which accounts for more than $90 \%$ childhood renal tumors. Pediatric non-Wilms renal tumors, such as renal PNETs and clear cell sarcomas are generally more aggressive and associated with a poorer prognosis [9]. Presentation is similar to other renal tumors; the classic triad of abdominal pain, palpable mass and hematuria is the most common manifestation $(84 \%$, $60 \%$ and $38 \%$, respectively) with fever and weight loss being infrequent symptoms [10]. No specific imaging features exist. The diagnosis is based on histopathology 


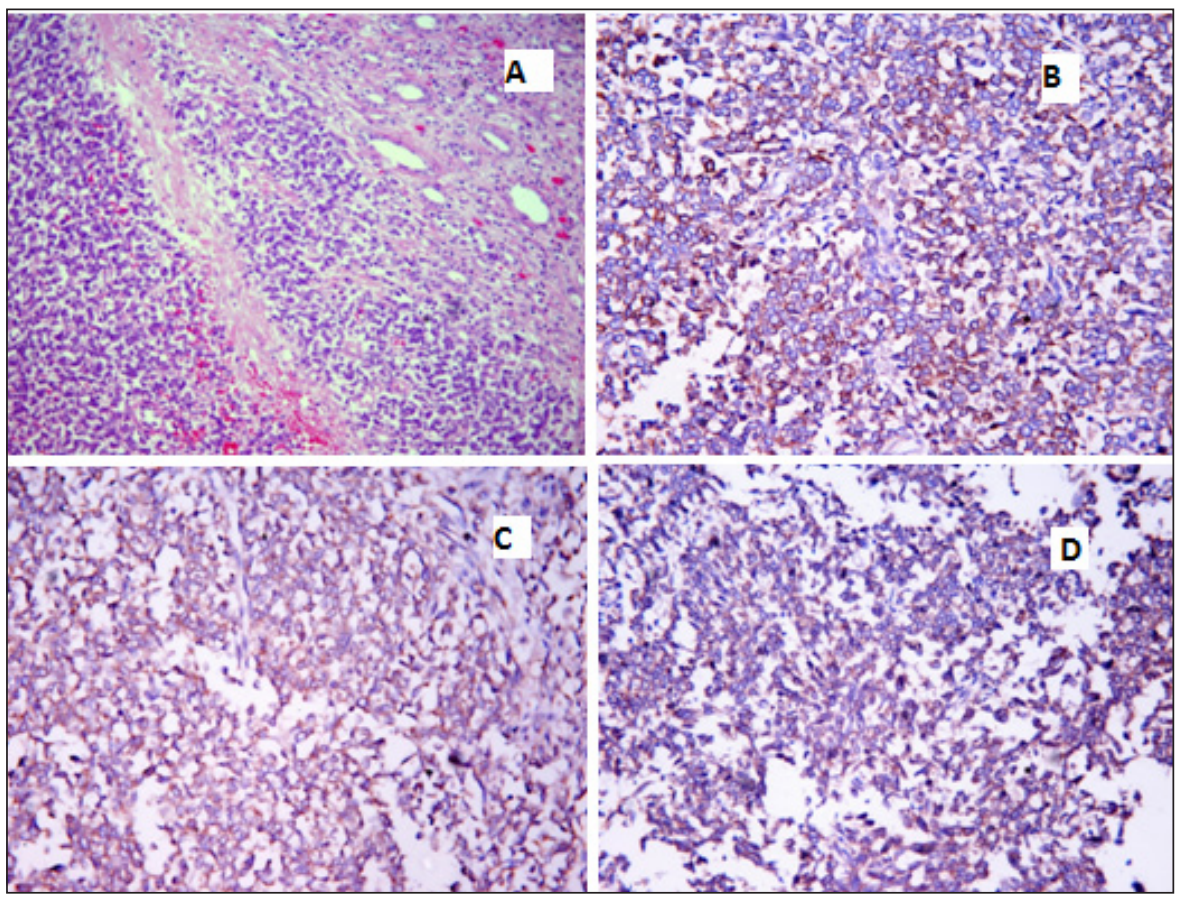

Fig. 2. Histopathology and Immunohistochemistry confirming the diagnosis of neuroectodermal tumor. A Hematoxylin-eosin stain revealing features of malignant round cell tumor- sheets of small, uniform, primitive malignant cells separated by fibrous bands. B, C, D are strongly immunopositive for CD 99 (MIC-2), synaptophysin and chromogranin respectively.

supported by immunohistochemistry- strong membranous CD99 positivity is a universal feature of PNETand the detection of chimeric transcripts. Approximately 90\% of ES/PNETs contain a EWS/FLI-1 fusion transcript that corresponds with the chromosome $t(11 ; 22)$ translocation; other cases maybe diagnosed on the basis of detection of other EWS-associated transcripts [9].

Patients with renal PNET have an aggressive disease course and have a poor prognosis especially if detected late, which is most often the case $[3,5,9,10]$. Thyavihally et al. [10] reported a $60 \%$ and $42 \%$ survival rate at 3 and 5 years, respectively. In another study of 40 renal PNET patients, almost half died within one year of diagnosis with a majority (70\%) developing metastatic disease [9].

The preferred treatment for renal PENT is surgical resection with adjuvant chemotherapy and radiotherapy treatment. The role of radiotherapy is not clear, but it may be advocated in locally advanced disease and involvement of Gerota's fascia [10]. Patients who have an associated thrombus undergo cavotomy or thrombectomy [9]. Most of the reported cases undergo (radical) nephrectomy, adjuvant chemotherapy (vincristine, ifos- famide, doxorubicin, cyclophosphamide and etoposide), radiotherapy or bone marrow transplant. Our patient was successfully managed with a radical nephrectomy, thrombectomy and adjuvant chemotherapy with vincristine, adriamycin, cyclophosphamide with alternating ifosfamide and etoposide.

\section{Conclusion}

To summarize, the diagnosis of renal PNETs should be considered in young adult patients who present with aggressive renal masses at initial presentations. Immunohistochemistry is of invaluable help for making a definite diagnosis. Despite its tendency to locally invade tissue, form venous tumor thrombi and metastasise, good outcomes can be achieved by a multimodality therapeutic strategy. 


\section{References}

1 Jimenez RE, Folpe AL, Lapham RL, Ro JY, O'Shea PA, Weiss SW, Amin MB: Primary Ewing's sarcoma/primitive neuroectodermal tumor of the kidney: a clinicopathologic and immunohistochemical analysis of 11 cases. Am J Surg Pathol 2002;26:320-327.

2 Takeuchi T, Iwasaki H, Ohjimi Y, Ohshima $\mathrm{K}$, Kaneko Y, Ishiguro M, Hiratsuka Y, Sakamoto K, Kikuchi M: Renal primitive neuroectodermal tumor: a morphologic, cytogenetic, and molecular analysis with the establishment of two cultured cell lines. Diagn Mol Pathol 1997;6:309-317.

3 Karnes RJ, Gettman MT, Anderson PM, Lager DJ, Blute ML: Primitive neuroectodermal tumor (extraskeletal Ewing's sarcoma) of the kidney with vena cava tumor thrombus. J Urol 2000;164:772.
4 Ng AW, Lee PS, Howard RG: Primitive neuroectodermal kidney tumour. Australas Radiol 2004;48:211-213.

5 Thomas JC, Sebek BA, Krishnamurthi V: Primitive neuroectodermal tumor of the kidney with inferior vena cava and atrial tumor thrombus. J Urol 2002;168:1486-1487.

6 Pomara G, Capello F, Cutanno MG, Rappa F, Morelli G, Mancini P, Selli C: Primitive neuroectodermal tumor (PNET) of the kidney: a case report. BMC Cancer 2004;4:3.

7 Parham DM, Roloson GJ, Feely M, Green DM, Bridge JA, Beckwith JB: Primary malignant neuroepithelial tumors of the kidney: a clinicopathologic analysis of 146 adult and pediatric cases from the National Wilms' Tumor Study Group Pathology Center. Am J Surg Pathol 2001;25:133-146.
8 Chu WC, Reznikov B, Lee EY, Grant RM, Cheng FW, Babyn P: Primitive neuroectodermal tumour (PNET) of the kidney: a rare renal tumour in adolescents with seemingly characteristic radiological features. Pediatr Radiol 2008;38:1089-1094.

9 Ellinger J, Bastian PJ, Hauser S, Biermann K, Müller SC: Primitive neuroectodermal tumor: rare, highly aggressive differential diagnosis in urologic malignancies. Urology 2006;68:257-262.

10 Thyavihally YB, Tongaonkar HB, Gupta S, Kurkure PA, Amare P, Muckaden MA, Desai SB: Primitive neuroectodermal tumor of the kidney: a single institute series of 16 patients. Urology 2008;71:292-296. 